\title{
Developing a survey of barriers and facilitators to recruitment in randomized controlled trials
}

\author{
Geetinder Kaur ${ }^{1,2^{*}}$, Rosalind L Smyth ${ }^{3}$ and Paula Williamson ${ }^{2}$
}

\begin{abstract}
Background: Recruitment to randomized controlled trials is known to be challenging. It is important to understand and identify predictors of good or poor accrual to a clinical trial so that appropriate strategies can be put in place to overcome these problems and facilitate successful trial completion. We have developed a survey tool to establish the recruitment experience of clinical teams regarding facilitators and barriers to recruitment in a clinical trial and describe herein the method of developing the questionnaire.

Methods: A literature search was conducted to identify studies that have explored facilitators and barriers to recruitment, and a list of potential factors affecting recruitment to a clinical trial was generated. These factors were categorized in terms relating to the (i) trial, (ii) site, (iii) patient, (iv) clinical team, (v) information and consent and (vi) study team. A list was provided for responders to grade these factors as weak, intermediate or strong facilitators or barriers to recruitment.

Results: A web-based survey questionnaire was developed. This survey was designed to establish the recruitment experience of clinical teams with regard to the perceived facilitators and barriers to recruitment, to identify strategies applied to overcome these problems, and to obtain suggestions for change in the organization of future trials. The survey tool can be used to assess the recruitment experience of clinical teams in a single/multicenter trial in any clinical setting or speciality involving adults or children either in an ongoing trial or at trial completion. The questionnaire is short, easy to administer and to complete, with an estimated completion time of 11 minutes.

Conclusions: We have presented a robust methodology for developing this survey tool that provides an evidence-based list of potential factors that can affect recruitment to a clinical trial. We recommend that all clinical trialists should consider using this tool with appropriate trial-specific adaptations to monitor and improve recruitment performance in an ongoing trial or conduct the survey at trial completion to gather information on facilitators and barriers to recruitment that can form the basis of interventions and strategies to improve recruitment to future clinical trials.
\end{abstract}

Keywords: Recruitment, Survey, Questionnaire, Clinical trials

\footnotetext{
* Correspondence: gkaur@liv.ac.uk

'Department of Women's and Children's Health, Institute of Translational Medicine, University of Liverpool, Institute of Child Health, Alder Hey

Children's Hospital, Eaton Road, Liverpool L12 2AP, UK

${ }^{2}$ Department of Biostatistics, Faculty of Health and Life Sciences, University of

Liverpool, Brownlow Street, Liverpool L69 3GS, UK

Full list of author information is available at the end of the article
} 


\section{Background}

Recruitment to randomized controlled trials is known to be challenging. However, effective and timely recruitment of appropriate and adequate numbers of research participants is essential for the successful completion of a trial and generation of valid results.

Prolonged or inefficient recruitment can have adverse scientific, economic and ethical consequences [1]. Failure to achieve the target sample size can lead to a reduction in the statistical power of a study. An underpowered study may report clinically important effects to be statistically non-significant and result in delay or non-implementation of a clinically effective intervention and delay in identification of non-effective interventions. Prolonged recruitment results in increased time or cost extensions and may result in premature termination of trials. Studies that terminate prematurely or fail to reach adequate statistical power raise 'ethical' concerns as trialists have exposed the participants to an intervention with uncertain benefit and may still be unable to determine whether the intervention does more harm than good at trial completion [2].

As under-recruitment is a common cause for trial failure, it is important to understand and identify the predictors of good or poor accrual to a clinical trial so that appropriate strategies can be put in place to overcome these problems and facilitate successful trial completion.

Several studies have examined recruitment experience from a number of perspectives. There are reports by trialists describing their recruitment experience, methods and strategies [3-10]. There are reports on recruitment and participation of under-represented populations such as minorities [11,12], adolescents, and young adults in cancer trials [13]. Studies have tried to assess parents' or families' reasons for participation or non-participation in trials [14-16], and there are several reports of surveys and interviews with parents or patients investigating the same [17-24].

Surveys and interviews with clinical teams have investigated reasons for considering patients unsuitable for a trial [25], reasons for not entering eligible patients [26], and difficulties with recruitment to the trial $[27,28]$. Caldwell et al. [29] conducted focus group discussions with sixteen pediatricians and five trainees from a pediatric teaching hospital to evaluate pediatricians' attitudes toward participation of children in randomized controlled trials and to identify potential barriers to participation. A number of studies have explored barriers to trial participation from patients' and clinicians' perspectives. Systematic reviews of studies [30-32] reporting barriers to participation in cancer trials have identified various patient and clinician-related barriers. Fayter et al. [33] conducted a systematic review to investigate the barriers, modifiers and benefits of participation in randomized controlled trials of cancer therapies as perceived by health care providers or patients and identified system-related or organizational barriers, trial design-related and health care provider barriers. Twenty-five studies explored barriers to participation from the health care perspective, with eight studies investigating recruitment to specific trials and seventeen studies investigating attitudes to trials in general. However, the authors concluded that the studies were of poor methodological quality and identified threats to internal validity in terms of potential for selection bias, non-justification of sample size, lack of reliability and validity of research instrument, and problems of data collection. None of the included surveys in this systematic review provided a comprehensive list of facilitators and barriers to recruitment.

Cook et al. [34] conducted a survey to explore the experiences, beliefs and practices of Critical Care Trials Groups regarding the effectiveness, feasibility and ethics of strategies to enhance enrollment and views on coenrollment of critically ill children and adults into one or more clinical studies. Fernandez et al. [35] conducted a trial-specific survey to explore the physicians' and parents' barriers to enrollment in the Children's Oncology Group's study of very low risk Wilm's tumor. Spaar et al. [36] conducted a postal survey among recruiting physicians in a multicenter trial of respiratory rehabilitation in patients with chronic obstructive pulmonary disease to identify and weigh barriers to recruitment to the trial. The survey questionnaire comprised barriers identified in literature that were applicable to the trial and concerns raised by recruiting physicians during the recruitment process. Studies [25-28,35] examine barriers to recruitment in the context of a specific trial or a specific population, and the survey questionnaires have been developed as trial or speciality specific [34]. Spaar et al. investigated some general barriers to recruitment as well, but not comprehensively, and recruitment facilitators were not identified. We have developed a survey instrument that can be used to investigate the experience of clinical teams with regard to both facilitators and barriers to recruitment to a single/multicenter clinical trial in any clinical setting or speciality. The survey questionnaire is evidence-based and has the potential to explore the generic factors affecting recruitment to a clinical trial with the scope of adding trial- and speciality-specific questions, thus providing a reliable tool and systematic approach to recognition and management of recruitment problems. To the best of our knowledge, there is no such existing recruitment survey tool, and we describe herein the method of developing this survey questionnaire.

\section{Methods \\ Survey design}

The survey has been designed as an online questionnaire to be completed by study teams involved with 
recruitment to a trial. The process of developing the questionnaire is presented in Figure 1. The survey is divided into four main sections to collect information about the site and study role of the responders, the perceived facilitators and barriers to recruitment, strategies applied to overcome the problems and suggestions for changes in organization of future trials.

Free-text space is provided for participants to enter information on their site or center of recruitment, their role in the study, whether they opened to recruitment, and their duration and period of involvement if they were not involved for the whole trial recruitment period. It is possible to add questions for collecting further information on the estimated number of eligible patients, the numbers expected to be randomized, and the hospital policy on recruitment to the study. Skip logic can be applied to direct questions selectively to responders depending on their response to previous questions so that they may skip questions that do not apply to them. The second section provides the survey participants with preformed lists of potential factors affecting recruitment that could act as facilitators or barriers to recruitment.

Defined the aims and scope of the survey and population of interest

Compiled content of the questionnaire: using evidence based list of potential factors affecting recruitment and providing a mix of open and closed questions

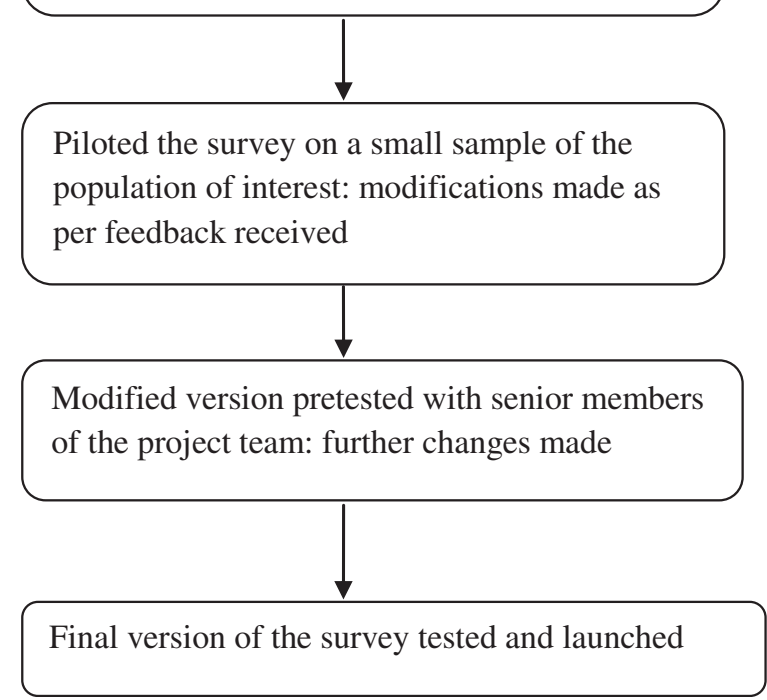

Figure 1 Process of developing the questionnaire.

\section{Writing the questionnaire}

A list of potential facilitators and barriers affecting recruitment to randomized controlled trials was made from a review of existing literature on the subject. A literature search on Medline using the search terms 'recruitment,' 'enrolment' combined with the AND connector to search terms for 'clinical trials' and 'randomised controlled trials' identified the major reviews on the subject that were used to develop the list of factors.

The reviews used to design the survey questionnaire are briefly described below. The process of selecting and classifying the factors for inclusion in the survey questionnaire is presented in Table 1. The Health Technology Assessment (HTA) report, 'Factors that limit the quality, number and progress of randomized controlled trials' by Prescott et al. [37] reported patient and clinician barriers to participation in randomized controlled trials. The authors of that report conducted a systematic review of studies that reported problems related to recruitment of clinicians and patients to clinical trials and identified the important barriers.

The HTA report 'Recruitment to randomised trials: strategies for trial enrolment and participation study: The STEPS study' aimed to identify the factors associated with good and poor recruitment to multicenter trials (38). They conducted an epidemiological review (The STEPS study Part A) of a cohort of trials funded by the Medical Research Council (MRC) and the National Health Service Health Technology Assessment (NHS HTA) program between January 1994 and December 2002. They tested hypotheses of factors for association with recruitment success in the cohort of multicenter randomized controlled trials included in the review, described patterns of recruitment and reported trialists' perceptions of factors associated with good or poor recruitment. The study also reported the reasons for delay in recruitment and early and late participant recruitment problems in the included cohort of trials based on the trialists' reports submitted to the funding bodies. The STEPS study (Part B) reported case studies of trials that recruited successfully and had particularly interesting lessons for recruitment. This aim of this part of the study was to gain role specific and location specific insights to the four included trials by interviewing 45 individuals in total across the four trials with different internal perspectives. Four key stages of a trial that may affect recruitment were identified: 1) foundation work involving engagement of collaborators, establishing scientific rigor, funding and financial considerations, 2) recruitment processes, 3) delivery of care and 4) delivery of research. Common factors in the success of the included trials were reported based on analysis of themes identified in the four key stages and from the responses of the interviewees. Toerien et al. [39] 
Table 1 Deriving the factors affecting recruitment from facilitators and from barriers described in literature

\section{Prescott et al. [37]}

Barriers to participation in clinical trials: patient and clinician barriers

\section{Barriers}

Patient barriers

Additional demands of the randomized controlled trials (RCT) on the patient

Additional procedures, additional appointments, time pressures, venepuncture, inpatient hospital stays, discomfort from medical procedures, length of study, worry about experimentation, uncomfortable procedures, travel and travel costs, extra costs

Patient preference for a particular treatment

Patients not wanting to change medication, not to take placebo, not to take experimental medication, not to take any medication, patient request for a specific intervention, strong patient preference for one treatment option

Aversion to treatment choice by random allocation

Worry about uncertainty

Efficacy of treatment on offer is unproven, distrust of hospital or medicine, fear of unknown

\section{Concerns about information and consent}

Amount of information provided to research participants, wording of information, complexity of information provided, different forms of information presentation: written /verbal/video, limited reading skills and English not being the primary language, clinicians' experience, difficulty in giving information, worry about level of information required and that information may be frightening, consent procedure barrier to recruitment

\section{Clinician barriers}

Time constraints

Time pressures from usual clinical practice, time demands of recruitment and follow-up

Staffing and training

Lack of trained staff, no additional support, lack of research experience in clinicians, lack of available support staff

\section{Rewards and recognition}

\section{Economic incentives}

Impact on doctor patient relationship fear of Adverse effect on doctorpatient relationship, perceived conflict in their role as clinicians and researchers

\section{Concern for patients}

Concern about treatment toxicity, side effects, burden of trial for patients including travel distance and costs, reluctance to recruit severely ill patients

Problems in complying with the protocol
Classification

Patient level factors

Patient level factors

Patient level factors

Information and consent related factors

Patient level factors

Clinical team factors

Clinical team factors

Clinical team factors

Excluded

Clinical team factors

Clinical team factor

Trial level factor
Factor derived

Additional trial investigations

Additional travel and extra costs

Duration of trial and follow- up

Patients'/parents' preference for a particular treatment

Patients'/parents' attitudes towards taking experimental medicine or placebo

Treatment choice by random allocation

Patients'/parents' concerns about side effects of new drug

Amount and complexity of trial

information provided

Clarity in presentation of trial information

Experience and training of clinical team seeking consent

Social and emotional dynamics of trial discussion

Consent rate

Language or cultural barrier

Difficulty in approaching

patents for consent

Clinical workload

Research experience of clinical team

Availability of designated research team

Availability of research staff out of hours

Presence of designated research nurse/ practitioner

Information available from the

Chief Investigator

Clinician attitude to involving patients in research

Clinician preference for a particular treatment

Study protocol compared to clinical practice 
Table 1 Deriving the factors affecting recruitment from facilitators and from barriers described in literature (Continued)

\section{Campbell et al. [38]}

Hypothesis of factors tested for association with recruitment success

Trials with complex trial design do not recruit as well as simple trials Less well-funded trials do not recruit well

Trials without dedicated trial management expertise do not recruit as well as those with trial management expertise

Trial with multidisciplinary input recruit better than those that do not have this input

Trials with consumer involvement recruit better than those that do not

Trials that have a successful pilot phase recruit better than those that do not have a pilot phase

Trials that have dedicated paid local coordinators recruit better than those that do not

Cancer trials recruit better than non cancer trials

Drug trials recruit better than non-drug trials

Trials funded through a response-mode funding have different recruitment rates to those funded through a commissioned process

Reasons for delays in recruitment to the included cohort of trials

Problems with central staff, local research staff, internal problems (for example, staff)

Local clinical arrangements, merging/reorganization of trusts, major relocation of services, department policies

Funding issues

Delays in ethical clearance

Research and Development (R\&D) delays,

time delay since grant application

Delays in supply of drug/placebo

Adverse publicity about medical research, external problem

(for example, publicity)

Setting up general practitioner (GP) practices took

longer than anticipated

Simultaneous other local research projects, competing research, conflict with other trials

Delays due to changes in data legislation, changes in technology

Fewer eligible than expected, smaller percentage

agreeing to participate, recruitment targets too ambitious

Absence of perceived clinical equipoise

Issues with procedures/interventions, trial process too demanding

Complexity of trial design, trial methodology considered too complex

Conflicting workload pressures, long waiting lists, additional theatre time required

Language/written English difficulties

Treatment preferences

\begin{tabular}{|c|c|}
\hline Trial level factor & Trial design \\
\hline Trial level factor & Funding \\
\hline Trial level factor & Trial management \\
\hline Excluded & $\begin{array}{l}\text { Information available from the } \\
\text { Chief Investigator }\end{array}$ \\
\hline Excluded & $\begin{array}{l}\text { Information available from the } \\
\text { Chief Investigator }\end{array}$ \\
\hline \multirow[t]{3}{*}{ Trial level factor } & Previous feasibility assessment \\
\hline & Previous pilot trial \\
\hline & Trial management \\
\hline \multirow[t]{2}{*}{ Trial level factor } & Being a drug/cancer trial \\
\hline & Being a drug/cancer trial \\
\hline Trial level factor & Funding \\
\hline Site level factor & Number of trained staff \\
\hline Clinical team factor & Motivation of clinical team \\
\hline Site level factor & Local clinical arrangements \\
\hline Trial level factor & Funding \\
\hline Excluded & $\begin{array}{l}\text { Information available from the } \\
\text { Chief Investigator }\end{array}$ \\
\hline
\end{tabular}

Excluded

Trial level factor Chief Investigator

Publicity by the trial team

External publicity

Site level factor

Time to open up site

Site level factor

Competing local research projects

Excluded

Trial level factor

Site level factor

Trial level factor

Patient level factor

Trial level factor

Clinical team factor

Patient level factor

Patient level factor

Clinical team factor
Information available from the Chief Investigator

Lack of pilot/feasibility assessment

Recruitment target

Clinical equipoise

Additional trial investigations

Trial design

Clinical workload

Language or cultural barrier

Patients'/parents' preference for a particular treatment

Clinician preference for particular treatment 


\section{Table 1 Deriving the factors affecting recruitment from facilitators and from barriers described in literature (Continued)}

Research not considered as priority

No local access to intervention

Case studies of trials: common factors in the successes of part B trials

Facilitator

Important/interesting research question, topic important, urgent need for research, important question,

timely and managed to roll several questions into one study

Good design/good protocol, pragmatic study

Clinicians keen to recruit to trial

Drugs already tested, so easy to explain to patients

Did not demand extra effort from patients, Impact on practice running and costs minimized, minimizing work for health professionals

No competing trials for those centers/patients

Drugs not available outside the trial

Excellent trial management, trial units helpful, caring, annual meetings for all concerned,

role of trial steering group

Good planning and organization by Clinical Trials Support Unit (CTSU), CTSU responsive, efficient, central organization of many aspects of research

Good communication between trial team and clinicians,

flexibility of trial teams

Good public relations/feedback/updates

Good funding, National Health Service (NHS) funding

Trial run by good team/infrastructure, Principal Investigator (PI) well respected, PIs worked hard to keep collaborators on board, trial team communicative, responsive and alert to problems. Communication within team, between team and collaborating clinicians

Good trial team, good research assistants

Team worked hard at how to explain the study to patients

Role of research nurse

Study included everybody

\section{Toerien et al. [39]}

Study design, number of arms, control: active/placebo

Single/multicenter

Intervention: drug/surgery/allied/others

Funding source
Clinical team factor

Patient level factor

Classification

Clinical team factor

Trial level factor

Clinical team factor

Patient level factor

Patient level factor

Site level factor

Patient level factor

Trial level factor

Study team factor

Trial level factor

Trial level factor

Study team factors

Clinical team factor

Clinical team factor

Trial level factor

Trial level factor

Excluded

Trial level factor

Trial level factor
Perceived importance of research generally in clinical practice

Perceived importance of the particular research question

Intervention available only in the trial

Factor derived

Perceived importance of the particular research question

Trial design

Study protocol compared to clinical practice

Motivation of clinical team

Clinician attitude to involving patients in research

Familiarity with experimental treatment Additional trial demands

Competing local research projects Intervention available only in the trial Trial management

Communication and coordination among study team members at site

Trial publicity

Funding

Motivation of the study team at site

Research experience of PI and study team members at site

Communication and coordination among study team members at site

Communication and coordination between study team at site and Clinical Trials Unit (CTU)

Research experience of clinical team

Communication skills of clinical team

Presence of designated research nurse/ practitioner

Patient inclusion criteria

Trial design

Information will be present

Being a drug/cancer/surgical/____trial

Funding 


\begin{tabular}{|c|c|c|}
\hline \multicolumn{3}{|l|}{ Caldwell et al. [40] } \\
\hline \multicolumn{3}{|l|}{ Recruitment strategies } \\
\hline Novel trial designs & Trial level factor & Trial design \\
\hline \multirow[t]{2}{*}{ Recruiter differences } & $\begin{array}{l}\text { Information and } \\
\text { consent related factors }\end{array}$ & $\begin{array}{l}\text { Experience and training of doctors clinical } \\
\text { team seeking consent }\end{array}$ \\
\hline & & Senior doctors and nurses seeking consent \\
\hline Financial incentives for patients/participants & Excluded & $\begin{array}{l}\text { Monetary incentives not acceptable } \\
\text { for clinical research in United Kingdom }\end{array}$ \\
\hline \multirow[t]{3}{*}{ Methods of providing information } & $\begin{array}{l}\text { Information and } \\
\text { consent related factors }\end{array}$ & $\begin{array}{l}\text { Amount and complexity } \\
\text { of information provided }\end{array}$ \\
\hline & Patient level factor & Clarity in presentation of trial information \\
\hline & & Consent rate \\
\hline \multicolumn{3}{|l|}{ Recruitment strategies } \\
\hline Design changes & Trial level factor & Trial design \\
\hline Modification to the consent form or process & Patient level factor & Consent rate \\
\hline \multirow[t]{3}{*}{ Modification to the approach made to potential participants } & $\begin{array}{l}\text { Information and } \\
\text { consent related factors }\end{array}$ & $\begin{array}{l}\text { Amount and complexity } \\
\text { of information provided }\end{array}$ \\
\hline & & Clarity in presentation of trial information \\
\hline & & Senior doctors and nurses seeking consent \\
\hline Financial incentives for patients/participants & Excluded & $\begin{array}{l}\text { Monetary incentives not acceptable } \\
\text { for clinical research in United Kingdom }\end{array}$ \\
\hline Modification to the training given to recruiters & $\begin{array}{l}\text { Information and } \\
\text { consent related factors }\end{array}$ & $\begin{array}{l}\text { Experience and training of clinical team } \\
\text { seeking consent }\end{array}$ \\
\hline Greater contact between trial coordinator and trial sites & Trial level factor & Trial management \\
\hline
\end{tabular}

reviewed the recruitment and retention rates in randomized controlled trials published in six major journals between July and December 2004 and investigated the association of these rates with trial characteristics such as study size, number of arms, single/multicenter, treatment focus (drug/surgery/allied/others), active/placebo control, time to assessment and type of funding. The Cochrane systematic review on strategies to improve recruitment to randomized controlled trials [2] identified 45 randomized and quasi-randomized controlled trials of interventions directed at potential participants or clinicians, which aimed to improve recruitment of participants to clinical trials. These interventions were divided into six categories: design change, modification to the consent form or process, modification to the approach made to potential participants, financial incentives for participants, modification of training given to recruiters and greater contact between trial coordinator and trial sites. The systematic review of strategies for increasing recruitment to randomized controlled trials by Caldwell et al. [40] looked at the effect of recruitment interventions such as novel trial designs, recruiter differences, incentives and different methods of providing trial information on recruitment success in randomized clinical trials.
From the facilitators and barriers reported in the above studies $(37,38)$ and the potential factors and interventions tested for association with recruitment success $(2,38-40)$, a list of potential factors affecting recruitment was generated by classifying the facilitators and barriers into various categories. This process is presented in Table 1 . The factors that were generic and expected to operate commonly at all sites were classified as 'trial level factors'. These included factors such as funding for the trial, trial design, choice of patient inclusion criteria, type of intervention, previous pilot/feasibility assessment, perception of clinical equipoise, publicity about the trial, trial management, etcetera The factors which could operate differentially between sites were classified as 'site level factors' and included factors such as time to open up site, recruitment target, local clinical arrangements, number and availability of trained staff, competing research projects and local research culture to list a few. We excluded factors for which objective information is available such as delays in ethical clearance, research and development delays, and problems with supply of investigational drug/placebo etcetera The various facilitators and barriers relating to patients' and clinicians' participation in clinical trials, as 
described in the above studies were listed under 'patient related and clinical team related factors'. The factors related to providing information to patients and seeking consent such as amount and complexity of trial information, clarity in presentation of trial information, time and setting of consent seeking and role and seniority of person seeking consent were categorized separately as information and consent related factors'. Lastly, the study team factors such as motivation and research experience of study team, communication and coordination between research teams were presented. Each category formed a separate question in the survey questionnaire to help the participants think through the issues arising during recruitment to the trial.

This section of the survey could be designed to elicit only barriers, only facilitators, or both barriers and facilitators to recruitment. In order to decrease the length of the survey and capture information on both facilitators and barriers in a common question, the factors were reworded such that they could apply both as a facilitator or barrier depending on whether they boosted or hindered recruitment respectively. The questions in this section were designed to obtain graded responses from ' -3 ' to ' +3 ' depending on whether the factor was perceived to be a strong $(-3)$, intermediate $(-2)$ or weak barrier $(-1), 0$ if thought to be not applicable and weak $(+1)$, intermediate $(+2)$, or strong facilitator $(+3)$.

Open-type questions were provided to obtain information on the various strategies applied to overcome the problems and for participants to express their reflective experiences and views on how trials could be organized differently in the future to improve recruitment.

\section{Pretesting/piloting the questionnaire}

The paper version of the questionnaire was sent for piloting to a small sample of five people. Three out of the five people (60\%) responded. The initial version had separate lists of facilitators and barriers and participants were asked to identify the top five in each list. Two out of the three respondents found the questionnaire lengthy, difficult to complete and it took them 35 to 40 minutes to do so. Some questions were thought to be ambiguous and there was a suggestion for use of computers to enhance the presentation and make it easier to complete.

After the pilot, the questionnaire was modified. The length of the questionnaire was reduced by combining the facilitators and barriers into a single list of factors that could be graded as either in the same question. Efforts were made to provide an evidence-based list of factors affecting recruitment while taking measures to keep the length of the survey and time of completion within reasonable limits. Factors for which objective information was believed to be available from other sources, such as delays in ethical or research and development approval or problems with supply of investigational drug/placebo were excluded from the questionnaire. However, free text space for additional comments was provided at the end for responders to note any issues not covered in the survey.

The factors were reworded so that they were simpler and clearer. An online version was created by using survey software (www.surveygizmo.com). The questions were arranged such that they had a logical flow. Each category of factors was arranged as small separate sections on a webpage for better presentation and ease of completion. The participants could easily navigate forward and backward to revisit a section if they needed to and the completion time was reduced to 10 to 15 minutes. The survey instrument (Additional file 1: Figure S1) has been used to investigate the recruitment experience of clinical teams in a large multicenter randomized controlled trial with children in the United Kingdom (MAGNETIC trial). The response rates are presented here, but the results of the survey for identified facilitators and barriers, the strategies applied to overcome these barriers and the suggestions for better organization of trials to improve recruitment, will be published separately. The MAGNETIC trial was a large multicenter, randomized, double-blind placebo-controlled trial evaluating the role of nebulized magnesium in acute severe asthma in children. It recruited over 500 children over a period of 26 months from 30 sites across the United Kingdom. The recruitment experience varied across sites and the opportunity to gather information about this study was believed to be important. There were three other sites that had opened to recruitment but did not recruit any patients and four others where efforts were made to set up the trial but did not open to recruitment. The survey was sent to all 37 sites as recruitment problems and perspectives of clinical teams were envisaged to be different at different sites. The names and contact email addresses of the potential responders were obtained from the delegation logs. This included all clinical team members including the Principal Investigators (PIs), research nurses (RNs),medical practitioners, nursing staff and nurse practitioners, who were delegated to be involved with recruitment to the trial. The survey was emailed to 522 contacts at all 37 sites. Since contact email addresses were expected to change over the course of the trial, especially for the various clinical staff, we selectively pursued responses from the Principal Investigator and at least one research nurse at each site.

\section{Results and discussion \\ Results}

The first section was designed to collect information about the responder's study role, site of recruitment and 
period and duration of his or her involvement in the trial. Personal information such as name was not collected; instead, each survey participant was assigned a unique identification code for purposes of data collection and analysis. Information on sites and study role was also used to examine representation in responses.

The second section provided a comprehensive list of factors affecting recruitment and the survey participants were asked to rate each factor from ' -3 ' to ' +3 ' as described. Each factor could be assigned only one score. This question was designed in this format to enable us to deduce the most commonly identified strong barriers and facilitators and also to enable us to calculate average scores for each factor. The last section had open-ended questions to gain information on the interventions applied and to collate reflective experiences and suggestions of the study team to improve recruitment with space for additional comments.

The project team sent initial invitations that described the aims and objectives of the survey, provided information about the survey, and requested participation in the survey to the potential participants. The email provided the link to the online questionnaire, stated that participation in the survey was voluntary, and that consent would be assumed by the person completing the questionnaire. The responders were reassured that no personal information such as names would be collected, no site will be identified in any publication and confidentiality of data will be maintained.

A total of 169 complete responses were obtained. We achieved a PI and one research nurse response per site from $86 \%$ and $94 \%$ of sites respectively. The response rates for PIs and RNs (one per site) are summarized in Table 2.

One hundred percent of the responders completed all questions ranking the factors affecting recruitment as facilitators or barriers. Of those responders 108 of 169 (64\%) responded to the open question on interventions or strategies applied to overcome the barriers identified. Free-text suggestions were obtained from 115 of the 169 (68\%) respondents on future reorganization of the trial to improve recruitment.

Table 2 Response rates for survey of facilitators and barriers to recruitment to the MAGNETIC trial

\begin{tabular}{lll}
\hline Sites $(\mathbf{n}=\mathbf{3 7})$ & $\begin{array}{l}\text { Principal } \\
\text { investigators } \mathbf{n}(\%)\end{array}$ & $\begin{array}{l}\text { Research } \\
\text { nurses n (\%) }\end{array}$ \\
\hline Sites that recruited $(\mathrm{n}=30)$ & $28(93 \%)$ & $28(93 \%)$ \\
$\begin{array}{l}\text { Sites that opened but did not } \\
\text { recruit }(\mathrm{n}=3)\end{array}$ & $2(67 \%)$ & $1(100 \%)^{a}$ \\
Sites that never opened $(\mathrm{n}=4)$ & $2(50 \%)$ & $1(100 \%)^{a}$ \\
Overall response & $32(86 \%)$ & $30(94 \%)$ \\
\hline
\end{tabular}

${ }^{\mathrm{a}}$ Research nurse present at only one site.

\section{Discussion}

A survey is a systematic method of collecting data from a population of interest, usually through the use of a structured and standardized questionnaire [41]. The methods of conducting survey research can be interviews, either face to face or via telephone, or using postal or electronic questionnaires. The advantages of a participant-completed questionnaire over an interview are that it is quicker and cheaper, avoids interviewer bias and allows respondents to record their responses privately even to sensitive issues. The disadvantages are that questions may be misunderstood or not fully answered by the respondent. There is a greater need to use closed questions to ensure consistency in the range of answers and for ease of analysis [42]. E-surveys offer a number of advantages over paper or telephone survey techniques in terms of less time and cost requirements, better accuracy in terms of fewer data transcription errors, faster creation and delivery, enhanced presentation and a higher response rates. The potential disadvantages include response bias resulting from unequal access to internet, issues of authenticity, data security and confidentiality, and respondent non-response or procrastination [43].

For these reasons, the recruitment survey questionnaire was developed as an online tool. Care was taken to avoid errors due to respondent misinterpretation of questions by phrasing the questions in a simple and clear manner. A mix of open and closed questions was provided to obtain accurate responses but also to provide respondents the freedom to express their views. Efforts were made to make the survey user-friendly by arranging the questions in a logical order and reducing the length of the survey. However, as for any other survey instrument the generation of useful results depends on a good response rate from a representative sample of the population of interest and obtaining true and accurate responses from participants. For the MAGNETIC trial, the recruitment survey questionnaire achieved a response from the Principal Investigator at $86 \%$ of the sites and at least one research nurse response from $94 \%$ of the sites. All the respondents completed the ranking of factors as facilitators or barriers, and free-text responses on interventions to overcome barriers and suggestions for future re-organization were obtained from $64 \%$ and $68 \%$ of the responders, respectively.

Recruitment to a clinical trial and its conduct is shaped by various internal and external forces including the shifting dynamics at sites because of changes in jobs and roles of staff including periodic turnover of trainee doctors every few months and change in policies at the hospital or trust level. Understanding the working of individual trials and of trial teams at various sites in a multicenter trial, with their unique challenges, as well as 
the responses of the research teams to these challenges, can provide important information that can be used to inform the design and conduct of future trials [38].

This survey questionnaire could be a very useful tool to investigate the recruitment experience of clinical teams and to identify facilitators and barriers to recruitment to a single or multicenter clinical trial in any clinical setting or speciality involving adults or children. It provides a common list of questions to participants at multiple sites and can be used to elicit the facilitators and barriers to trial participation in general, and can also be adapted and modified by adding trial specific questions and highlighting trial- and speciality-specific recruitment issues. It provides a mix of open and closed questions and allows for free-text space for participants to share their experiences and make reflective comments. It is designed to gather data from people with a range of responsibilities related to recruitment to the trial. It can be aimed at staff directly involved with recruitment but can also be extended to other staff that facilitate recruitment or are involved indirectly to gain an insight into their perspective on issues around recruitment to the trial. The survey can be easily sent to a large number of participants at the same time. It can be used to gauge role- and site-specific perceptions of the research team and can provide a detailed understanding of the various factors affecting recruitment in addition to providing information from other monitoring tools such as screening logs.

This survey tool was designed to be used at the end of the recruitment phase of a study to identify useful lessons for future research and to other trialists. It could be used however, with some modification, in the pre-trial phase to identify potential problems or in the early and middle recruitment phases when observed participation rate is lower than expected. During a trial, the study team will often contact under-recruiting sites to obtain information about problems encountered or will contact higher than average recruiting sites to identify facilitators. This tool would provide a more systematic approach to the collection and consideration of such information, ensuring that all evidence-based barriers and facilitators are reviewed by the site in their response, so that appropriate strategies can be implemented to overcome the problems identified. If the survey is to be undertaken during the recruitment phase to identify modifiable aspects of the process, factors such as the time taken to open the site and whether there was a previous feasibility or pilot study would not be relevant.

Since recruitment performance is usually variable at different sites, it can be used to investigate the various site-specific issues. This will not only provide a detailed understanding of the internal milieu of the trial but also provides the opportunity for comparison of responses between successful and non-successful sites. Identification of facilitators or barriers and strategies applied at sites with successful recruitment in comparison to less successful sites may highlight some modifiable differences, which can form the basis of interventions and strategies to boost recruitment to an ongoing clinical trial or provide useful lessons for designing and conducting future trials. The survey questionnaire has some potential limitations. Being a subjective tool, it is prone to responder misinterpretation, and the authors encourage trialists to pilot the questionnaire with a sample of their trial team prior to use to ensure consistent understanding of the listed factors. Though it has been designed to provide an evidence-based list of generic factors that affect recruitment to clinical trials, the authors would again encourage trialists to think about other anticipated or observed trialspecific issues and modify and adapt the questionnaire before use, taking into consideration the type of trial and the stage of recruitment. The length of the survey can be reduced further by excluding factors that are thought to be not relevant to a particular trial.

\section{Conclusions}

Recruitment to clinical trials is a common problem. Understanding the working of individual trials and identification of recruitment facilitators and barriers can provide important information to improve recruitment to the trial and to help with better design in future trials. We have developed a recruitment survey tool providing an evidence-based list of generic factors that can affect recruitment to a clinical trial. It can be used in any clinical setting or speciality involving adults or children and can be modified by adding trial-specific issues. Clinical trialists may identify other trial specific recruitment issues and make appropriate modifications. Piloting the survey questionnaire with the trial teams prior to use is advisable to ensure consistency in the understanding of terminology.

This survey tool is a potentially useful tool that can help trialists to approach recruitment problems in a systematic manner. It has been used successfully to elicit barriers and facilitators to recruitment in the MAGNETIC trial but would need further evaluation in other trials. If proved to be useful, we would recommend that clinical trialists consider using and adapting this tool to monitor and improve recruitment performance in an ongoing trial or to conduct the survey at trial completion to gather information on facilitators and barriers to recruitment. This information will form the basis of interventions and strategies to enhance recruitment to clinical trials and channelize resources effectively to counter the problems of under-recruitment in future clinical research. 


\section{Additional file}

Additional file 1: Figure S1. Recruitment survey.

\section{Abbreviations}

HTA: Health Technology Assessment; MRC: Medical Research Council; NHS: National Health Service.

\section{Competing interests}

The authors declare that they have no competing interests.

\section{Authors' contributions}

GK reviewed the literature, designed and developed the survey tool, and drafted the manuscript. RS contributed to survey design, participated in critical revision of the manuscript, and gave final approval of version to be published. PW conceived the initial idea, contributed to survey design, participated in critical revision of the manuscript and gave final approval of version to be published. All authors read and approved final manuscript.

\section{Acknowledgements}

\section{Funding for Authors}

GK was supported by the Medical Research Council North West Hub for Trials Methodology Research. Funding for preparation of manuscript was provided by the Medical Research Council North West Hub for Trials Methodology Research. The authors would like to thank the reviewers-Alison McDonald, Shaun Treweek and Haleema Shakur-for their helpful comments and suggestions.

\section{Author details}

${ }^{1}$ Department of Women's and Children's Health, Institute of Translational Medicine, University of Liverpool, Institute of Child Health, Alder Hey Children's Hospital, Eaton Road, Liverpool L12 2AP, UK. ²Department of Biostatistics, Faculty of Health and Life Sciences, University of Liverpool, Brownlow Street, Liverpool L69 3GS, UK. ${ }^{3}$ UCL Institute of Child Health, 30 Guilford Street, London WC1N 1EH, UK.

\section{Received: 25 March 2012 Accepted: 18 October 2012}

Published: 21 November 2012

\section{References}

1. Gul RB, Ali PA: Clinical trials: the challenge of recruitment and retention of participants. J Clin Nurs 2010, 19:227-233

2. Treweek S, Pitkethly M, Cook J, Kjeldstrøm M, Taskila T, Johansen M, Sullivan F, Wilson S, Jackson C, Jones R, Mitchell E: Strategies to improve recruitment to randomized controlled trials. Cochrane Database Syst Rev 2010, 4:MR000013.

3. Wardle K, Murphy D, Ireland L, Holbeck C, Davidson C, Wen LM, Rissel C: What we learnt-recruiting prenatal mothers to an RCT addressing the prevention of overweight in early childhood? Aust J Adv Nurs 2010, 28:41-45.

4. Finne E, Reinehr T, Schaefer A, Winkel K, Kolip P: Overweight children and adolescents-is there a subjective need for treatment? Int J Public Health 2009, 54:112-116.

5. Baines CJ: Impediments to recruitment in the Canadian National Breast Screening Study: response and resolution. Control Clin Trials 1984, 5:129-140.

6. Galbreath AD, Smith B, Wood P, Forkner E, Peters Jl: Cumulative recruitment experience in two large single-center randomized controlled clinical trials. Contemp Clin Trials 2008, 29:335-342.

7. Vollmer WM, Hertert S, Allison MJ: Recruiting children and their families for clinical trials: a case study. Control Clin Trials 1992, 13:315-320.

8. Heinrichs N, Bertram H, Kuschel A, Hahlweg K: Parent recruitment and retention in a universal prevention program for child behavior and emotional problems: barriers to research and program participation. Prev Sci 2000, 6:275-286.

9. Bailey JM, Bieniasz ME, Kmak D, Brenner DE, Ruffin MT: Recruitment and Retention of economically underserved women to a cervical cancer prevention trial. Appl Nurs Res 2004, 17:55-60.
10. Strunk R, Sternberg AL, Belt P, Caesar M, Chinn T, Gleason M, Hall A, Harden K, Kelly W, Madden N, Plunkett A, Shapiro G, Tata M, Natta MV, Wheeler B, Zeiger R: Recruitment of participants in the Childhood Asthma Management Program (CAMP): Description of Methods. J Asthma 1999, 36:217-237

11. Baquet CR, Commiskey P, Mullins D, Mishra Sl: Recruitment and participation in clinical trials: Socio-demographic, rural/urban, and health care access predictors. Cancer Detect Prev 2006, 30:24-33.

12. Nicholson LM, Schwirian PM, Klein EG, Skybo T, Murray-Johnson L, Eneli I, Boettner B, French GM, Groner JA: Recruitment and retention strategies in longitudinal clinical studies with low-income populations. Contemp Clin Trials 2011, 32:353-362.

13. Fern LA, Whelan JS: Recruitment of adolescents and young adults to cancer clinical trials- International comparisons, barriers and implications. Semin Oncol 2010, 37:e1-e8.

14. Peden V, Choonara I, Gennery B, Done H: Recruiting children to a clinical trial. Paediatr Perinat Drug Ther 2000, 4:75-78.

15. Wynn L, Miller S, Faughnan L, Luo Z, Debenham E, Adix L, Fish B, Hustace T, Kelly T, MacDermott M, Marasciulo J, Martin B, McDuffie J, Murphy M, Rackoff B, Reed C, Seaman P, Thomas G, Wang W: Recruitment of infants with sickle cell anemia to a Phase III trial: Data from the BABY HUG study. Contemp Clin Trials 2010, 31:558-563.

16. Mihrshashi S, Vukasin N, Forbes S, Wainwright C, Krause W, Ampon R, Mellis C, Marks G, Peat J: Are you busy for the next five years? Recruitment in the Childhood Asthma Prevention Study (CAPS). Respirology 2002, 7:147-151.

17. Weintraub J, Leske GS, Ripa LW, Levinson A: Recruitment of a clinical field trial population: reasons for nonparticipation. J Public Health Dent 1980, 40:141-145.

18. Sharp L, Cotton SC, Alexander L, Williams E, Gray NM, Reid JM: Reasons for participation and non-participation in a randomized controlled trial: postal questionnaire surveys of women eligible for TOMBOLA (Trial of Management of Borderline and other Low Grade Abnormal Smears). Clin Trials 2006, 3:431-442.

19. Driscoll KA, Johnson SB, Schatz DA, Haller MJ: Use of a precious resource: Parental decision making about using autologous umbilical cord blood in studies involving young children with type 1 diabetes. Contemp Clin Trials 2011, 32:524-529.

20. Smyth RMD, Duley L, Jacoby A, Elbourne D: Women's experiences of participating in the magpie trial: a postal survey in the United Kingdom. Birth 2009, 36:220-229.

21. Eiser C, Davies H, Jenney M, Glaser A: Mother's attitudes to the randomized controlled trial $(\mathrm{RCT})$ : the case of acute lymphoblastic leukaemia (ALL) in children. Child Care Health Dev 2005, 31:517-523.

22. Nabulsi M, Khalil $Y$, Makhoul J: Parental attitudes towards and perceptions of their children's participation in clinical research: a developing country perspective. J Med Ethics 2011, 37:420-423.

23. Cain MA, Mc Guinness C: Patient recruitment in paediatric clinical trials. Pract Diabetes Int 2005, 22:328-332.

24. Dolan LA, Sabesan V, Weinstein SL, Spratt KF: Preference assessment of recruitment into a randomized trial for adolescent idiopathic scoliosis. J Bone Joint Surg 2008, 90:2594-2605.

25. Hunt CJ, Shepherd LM, Andrews G: Do doctors know best? Comments on a failed trial. Med J Aust 2001, 174:144-146.

26. Taylor KM, Margolese RG, Soskolne CL: Physicians' reasons for not entering eligible patients in a randomized clinical trial of surgery for breast cancer. N Engl J Med 1984, 310:1363-1367.

27. Fairhurst K, Dowrick C: Problems with recruitment in a randomized controlled trial of counselling in general practice: causes and implications. J Health Serv Res Policy 1996, 1:77-80

28. Brooker C, Peters J, McCabe C, Short N: The views of nurses to the conduct of a randomised controlled trial of problem drinkers in an accident and emergency department. Int J Nurs Stud 1999, 36:33-39.

29. Caldwell PHY, Butow PN, Craig JC: Pediatricians' attitudes toward randomized controlled trials involving children. J Pediatr 2002, 141:798-803.

30. Ellis PM: Attitudes towards and participation in randomised clinical trials in oncology: a review of the literature. Ann Oncol 2000, 11:939e45.

31. Cox K, McGarry J: Why patients don't take part in cancer clinical trials: an overview of the literature. Eur J Cancer Care 2003, 12:114e22.

32. Tournoux C, Katsahian S, Chevret S, Levy V: Factors influencing inclusionof patients with malignancies in clinical trials. Cancer 2006, 106:258e70. 
33. Fayter D, McDaid C, Eastwood A: A systematic review highlights threats to validity in studies of barriers to cancer trial participation. J Clin Epidemiol 2007, 60:990-1001

34. Cook DJ, Blythe D, Rischbieth A, Hebert PC, Zytaruk N, Menon K, Erikson S, Fowler R, Heels-Ansdell D, O Meade M: Enrollment of intensive care unit patients into clinical studies: A trinational survey of researchers practices. Crit Care Med 2008, 36:2100-2105.

35. Fernandez CV, Li N, Mullen EA, Grundy PE, Perman EJ, Shamberger RC, Ehrlich PF, Dome JS: Barriers to enrolment of children in the children's oncology group study of very low risk Wilm's tumor: a report from the children's oncology group. J Pediatr Hematol Oncol 2011, 33:521-523.

36. Spaar A, Frey M, Turk A, Karrer W, Puhan MA: Recruitment barriers in a randomised controlled trial from the physicians' perspective: a postal survey. BMC Med Res Methodol 2009, 9:14.

37. Prescott RJ, Counsell CE, Gillespie WJ, Grant AM, Russell IT, Kiauka S, Colthart $\mathbb{R}$, Ross S, Shepherd SM, Russell D: Factors that limit the quality, number and progress of randomised controlled trials. Health Technol Assess 1999, 3:1-143.

38. Campbell MK, Snowdon C, Francis D, Elbourne D, McDonald AM, Knight R, Entwistle V, Garcia J, Roberts I, Grant A: Recruitment to randomised trials: strategies for trial enrolment and participation study. The STEPS study. Health Technol Assess 2007, 11:lii. ix-105.

39. Toerien M, Brookes ST, Metcalfe C, De Salis I, Tomlin Z, Peters TJ, Sterne J, Donovan JL: A review of reporting of participant recruitment and retention in RCTs in six major journals. Trials 2009, 10:52.

40. Caldwell PHY, Hamilton S, Tan A, Craig JC: Strategies for increasing recruitment to Randomised Controlled Trials: Systematic Review. PLoS Med 2010, 7:e1000368.

41. The Health Communication Unit (THCU) Resource Library. http://www.thcu.ca/ resource_db/pubs/729877940.pdf.

42. Bruce N, Pope D, Stanistreet D: Surveys. In Quantitative methods in Health Research: a practical interactive guide. 1st edition. London: John Wiley; 2008:129-191.

43. Anderson T, Kanuka T: Surveys. In E-Research: Methods, strategies and issues. 1st edition. London: Allyn and Bacon; 2003:146-172.

doi:10.1186/1745-6215-13-218

Cite this article as: Kaur et al.: Developing a survey of barriers and facilitators to recruitment in randomized controlled trials. Trials 2012 13:218.

\section{Submit your next manuscript to BioMed Central and take full advantage of:}

- Convenient online submission

- Thorough peer review

- No space constraints or color figure charges

- Immediate publication on acceptance

- Inclusion in PubMed, CAS, Scopus and Google Scholar

- Research which is freely available for redistribution 